\title{
Variables que determinan las exportaciones de un recurso natural no renovable: caso del gas natural licuado peruano, periodo 2010-2016
}

\author{
Variables that determine the exports of a non-renewable natural resource: case of the peruvian \\ liquefied natural gas, period 2010-2016
}

\section{Oscar Fernando Navarro Angeles ${ }^{1}$}

\section{Resumen}

El valor de las exportaciones de gas natural licuado del Perú ha presentado un comportamiento fluctuante en el período 2010-2016, evidenciado por su tasa de crecimiento, que ha influenciado en su participación en el valor de las exportaciones tradicionales en el rubro petróleo y gas natural. Se ha determinado el comportamiento y relación entre las variables relevantes que han influenciado en las exportaciones del gas natural licuado. La demanda de España, México, Japón y Corea del Sur, ha sido importante en el comportamiento fluctuante, creciente y decreciente del valor de las exportaciones de gas natural licuado del Perú.

Palabras clave: variables; exportaciones; importaciones; recurso; gas natural licuado; Perú; precio; demanda mundial; consumo; mercado.

\begin{abstract}
The value of Peruvian liquefied natural gas exports has shown a fluctuating behavior in the period 2010-2016, evidenced by its growth rate, which has influenced its share of the value of traditional exports in the Oil and Natural Gas sector. The behavior and relationship between the relevant variables that have influenced exports of liquefied natural gas have been determined. Demand from Spain, Mexico, Japan and South Korea has been important in the fluctuating, increasing and decreasing behavior of the value of Peruvian liquefied natural gas exports.
\end{abstract}

Keywords: variables; exports; imports; resource; liquefied natural gas; Peru; price; world demand; consumption; market.

\section{Introducción}

La exportación de Gas Natural Licuado (GNL) del Perú se inició en el segundo trimestre del 2010, alcanzado el valor Free on boar (FOB) de US\$283,5 millones (Gerencia Central de Estudios Económicos-BCRP. s.f.) en el referido año, siendo los principales países de destino (The Observatory of Economic Complexity [OEC], s.f.: España (31,03\%), EEUU (20,17\%), Corea del Sur (13,42\%), Brasil (11,01\%), México $(8,06 \%)$ y China $(7,09 \%)$. En los siguientes años el valor aumentó a US\$ $1.371,8$ millones (2013) y luego cae a US\$ 523,4 millones (2016), Figura.1. En este último año los principales países de destino fueron España (40,85\%), México (26,15\%), Corea del Sur $(10,21 \%)$, China $(8,76 \%)$ y Japón $(7,23 \%)$.

En cuanto a su participación en el valor de las exportaciones de Hidrocarburos del Perú, la exportación de GNL representó el
28,1\% en el 2011, pero luego cae al 23,7\% en el 2016 . En este último año la participación de las exportaciones de GNL en el valor de las exportaciones mineras e hidrocarburos del Perú solo representó el 2,18\%, a pesar de esta baja participación en el 2016, alcanzó el $4,72 \%$ en el 2013 (US\$ 1.371,8 millones), lo que muestra la posibilidad de alcanzar valores similares en los siguientes años. 


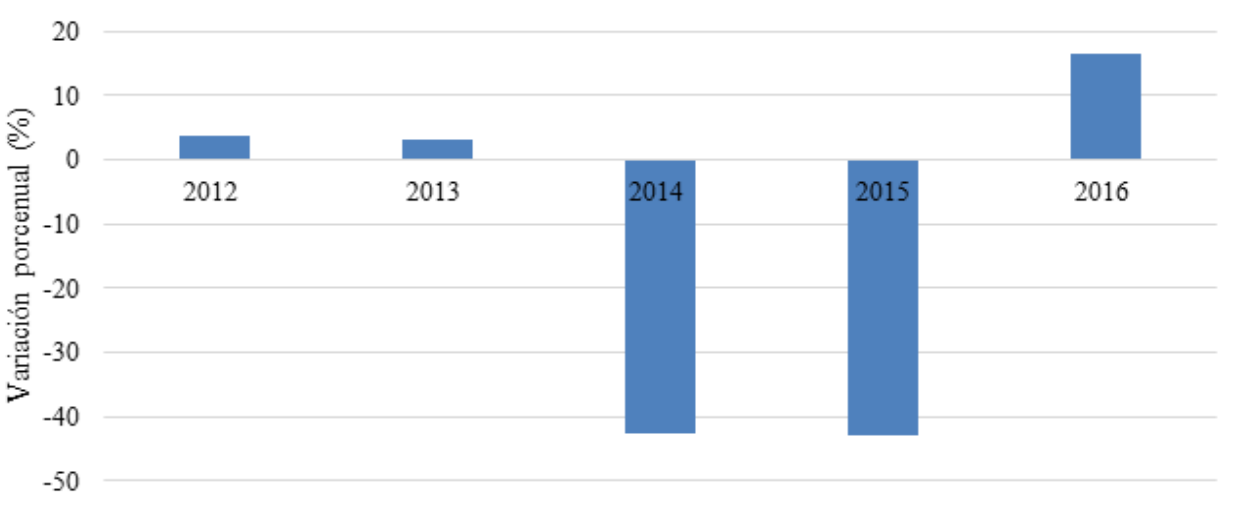

Figura 2. Variación porcentual del valor de las exportaciones de GNL del Perú, período 2012-2016

Fuente: Gerencia Central de Estudios Económicos. BCRP.

Durante el período de estudio (2010-2016) el valor de las exportaciones de GNL mostró un comportamiento fluctuante, evidenciado en su tasa de crecimiento anual (Figura 2), la cual ha oscilado entre $-42,9 \%$ (2015) y $16,5 \%$ (2016). Cabe indicar que la tasa de crecimiento en el 2011 fue de $352,7 \%$, esto debido a que las exportaciones de GNL se iniciaron en el 2010 con un valor de US\$283,5 millones y en el 2011 fue de US\$1.283,5 millones.

Estas fluctuaciones en el valor de las exportaciones se explicarían por el comportamiento del precio, el volumen exportado y la demanda de GNL por los principales países de destino, por lo que es importante identificar los factores que han influenciado en las referidas variables durante el período del estudio.

Debido a la relativa importancia de la contribución de las exportaciones de GNL en la disponibilidad de divisas para el país, que al 2015 existen reservas probadas de gas natural de 14,1 billones de pies cúbico (BCF) y reservas probables de 3,8 (BCF). Según el Observatorio Energético Minero [Osinergmin], s.f., que con las reservas probadas al 2016 se tiene un horizonte de abastecimiento mínimo de quince años para el mercado interno y externo, y dado el comportamiento fluctuante en el corto plazo y la tendencia decreciente del valor de las exportaciones de GNL en el último quinquenio, se tiene por objetivo general determinar la relación entre valor de las exportaciones de GNL del Perú con las siguientes variables relevantes de influencia: precio, volumen exportado y demanda de los principales países de destino (España, México, Corea del Sur, Japón y China) durante el período 2010-2016.

\section{Materiales y métodos}

Siendo el objetivo general del presente estudio determinar la relación existente del valor de las exportaciones de GNL del Perú con el precio, volumen exportado y demanda de los principales países de destino (España, México, Corea del Sur, Japón y China) durante el período 2010-2016, los métodos de investigación empleados han sido el Deductivo e Inductivo.
Método Deductivo: en base a la teoría económica, se buscó explicar las causas del comportamiento del valor de las exportaciones de GNL, para lo cual se identificó como variables explicativas: el precio, volumen de exportación de GNL del Perú, y la demanda de los principales países de destino.

Método Inductivo: en base al análisis de los datos históricos se buscó determinar el comportamiento de las variables relevantes y la existencia de correlación entre ellas (dependiente y explicativas).

La metodología empleada comprendió los siguientes pasos:

1. Para el estudio del comportamiento de las variables relevantes se utilizó series trimestrales y anuales provenientes de fuentes secundarias (Banco Central de Reserva del Perú, s.f.; BP Statistical Review of World Energy, 2017; Observatorio Energético Minero de Osinergmin, s.f.; The Observatory of Economic Complexity, S.f.). Asimismo, se analizó la evolución de las series de tiempo, lo que permitió determinar el comportamiento fluctuante a corto plazo, las tendencias a mediano plazo y las relaciones entre variables.

2. Para los estudios de correlación, que buscó establecer relaciones directas e inversas entre variables, se determinó el coeficiente de correlación entre las series. La metodología empleada permitió identificar las relaciones existentes entre las variables relevantes, para ello se utilizó las funciones estadísticas del Excel 2013.

\section{Resultados y discusión}

Evolución del valor de las exportaciones de GNL del Perú

Durante el período 2010-2016, el valor trimestral de las exportaciones de GNL del Perú mostró un comportamiento estacional en el cuarto trimestre de todos los años (con excepción del 2012), donde se observa tasas de crecimiento positivo (Figura 3) que coinciden con el inicio de la estación de invierno de los principales países de destino (España, México, Corea del Sur, China y Japón), países que en el 2016 demandaron el 93,2\% del valor de las exportaciones de GNL del Perú.

Debido a que el valor de las exportaciones de GNL es el resultado de la multiplicación del volumen de las exportaciones por el precio, su comportamiento ha sido influenciado por el volumen de las exportaciones, que 


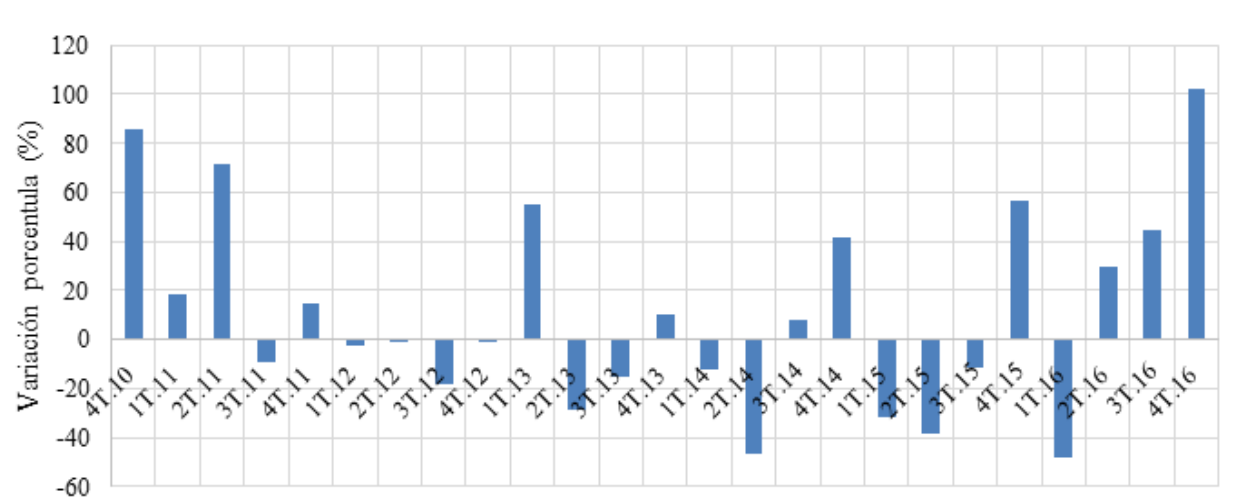

Figura 3. Variación porcentual del valor trimestral de las exportaciones de GNL del Perú, período 2010-2016

Fuente: Gerencia Central de Estudios Económicos. BCRP

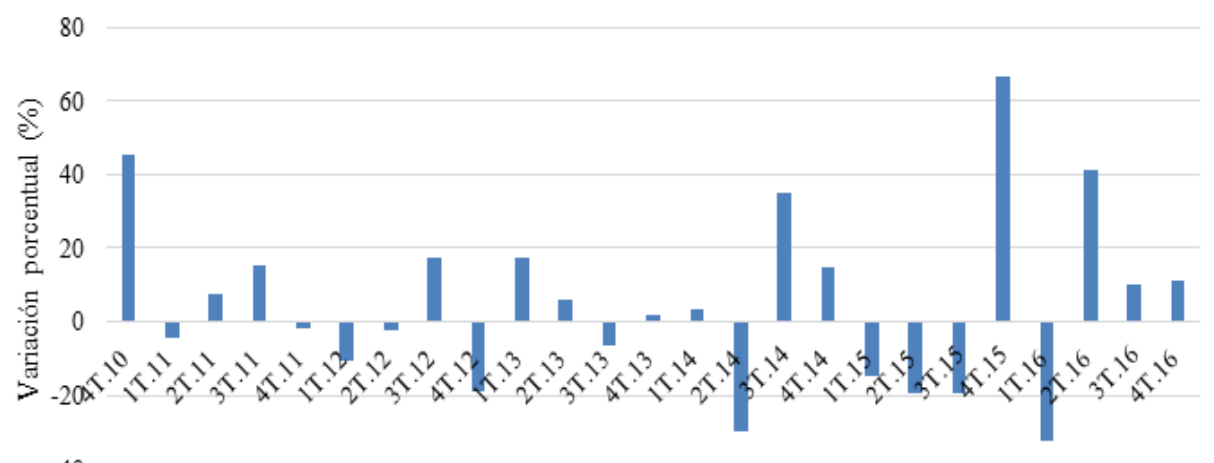

$-40$

Figura 4. Variación porcentual del volumen trimestral de las exportaciones de GNL del Perú, período 2010-2016

Fuente: Gerencia Central de Estudios Económicos. BCRP

mostró significativas tasas de crecimiento positivo en el cuarto trimestre de los años 2010, 2014, 2015 y 2016 (Figura 4), coincidiendo con el comportamiento del valor de las exportaciones.

En cuanto al valor trimestral de las exportaciones de GNL del Perú, mostró una tendencia creciente hasta el 1T2013, habiendo alcanzado su valor máximo de US\$ 460,4 millones. Luego muestra una tendencia decreciente hasta el 1T-2016, habiendo alcanzado un valor mínimo de US\$ 65,7 millones, y se recuperó hasta el último trimestre del 2016 (Figura 5).

Cabe notar que el valor promedio trimestral de las exportaciones de GNL fue de US\$ 336,83 millones en el periodo 2T-2011 al 1T-2014, y luego disminuyó a US\$ 124,1 millones en el período 2T-2014 al 4T-2016, lo que implicó una caída significativa de 63,16\%.

En cuanto al comportamiento del precio del GNL(Figura 6), mostró también un comportamiento estacional, pues se observó tasas de crecimiento positivo en el cuarto trimestre del período 2010-2016, con excepción del 2015. Asimismo, se observó tasas de crecimiento negativo en el tercer trimestre del período 2011-2014, pero en el 2014 también se observó tasas de crecimiento negativo en el primer y segundo trimestre, comportamiento que se mantiene en los años 2015 y 2016. Es decir, el comportamiento estacional del precio con tasas de crecimiento negativo se traslada del tercer trimestre del 20112014 al primer y segundo trimestre del 2014-2016.

En su relación con el valor de las exportaciones de GNL, la tasa trimestral de crecimiento negativo del precio influenció en la caída del valor de las exportaciones en los trimestres: 3T-2011, 3T2012, 2T-2013 y 1T-214, esto a pesar de la tasa de crecimiento positivo del volumen de las exportaciones observada en los referidos trimestres.

Si observamos el comportamiento anual, las caídas significativas del valor de las exportaciones de GNL del Perú en el 2014 y 2015 se han debido a la caída del volumen de las exportaciones y del precio (Tabla 1). En relación al 2012, 2013 y 2016, aun cuando el volumen de las exportaciones y el precio tuvieron tasas de crecimiento con signos opuestos, siempre la tasa de crecimiento positivo fue mayor al negativo, produciendo como efecto neto tasas de crecimiento positivo en el valor de las exportaciones.

Tabla 1. Variación porcentual del valor, volumen y precio de las exportaciones de GNL del Perú, período 2010-2016

\begin{tabular}{cccc}
\hline & $\begin{array}{c}\text { Variación } \\
\text { porcentual del } \\
\text { valor de las } \\
\text { exportaciones (\%) }\end{array}$ & $\begin{array}{c}\text { Variación } \\
\text { porcentual del } \\
\text { volumen de las } \\
\text { exportaciones (\%) }\end{array}$ & $\begin{array}{c}\text { Variación } \\
\text { porcentual del } \\
\text { precio (\%) }\end{array}$ \\
\hline 2011 & 352,7 & 148,8 & 82 \\
2012 & 3,7 & $-2,6$ & 6,4 \\
2013 & 3,1 & 9,4 & $-5,8$ \\
2014 & $-42,7$ & $-3,5$ & $-40,6$ \\
2015 & $-42,9$ & $-12,3$ & $-34,9$ \\
2016 & 16,5 & 18,5 & $-1,6$ \\
\hline
\end{tabular}

Fuente: Gerencia Central de Estudios Económicos. BCRP

\section{Evolución del volumen de las exportaciones de GNL del Perú}

Las exportaciones de GNL del Perú se iniciaron en el 2T2010, exportándose en el referido año 3,605 millones de $\mathrm{m}^{3}$. En los siguientes años, el volumen de las exportaciones anuales tuvo un comportamiento fluctuante en el corto 


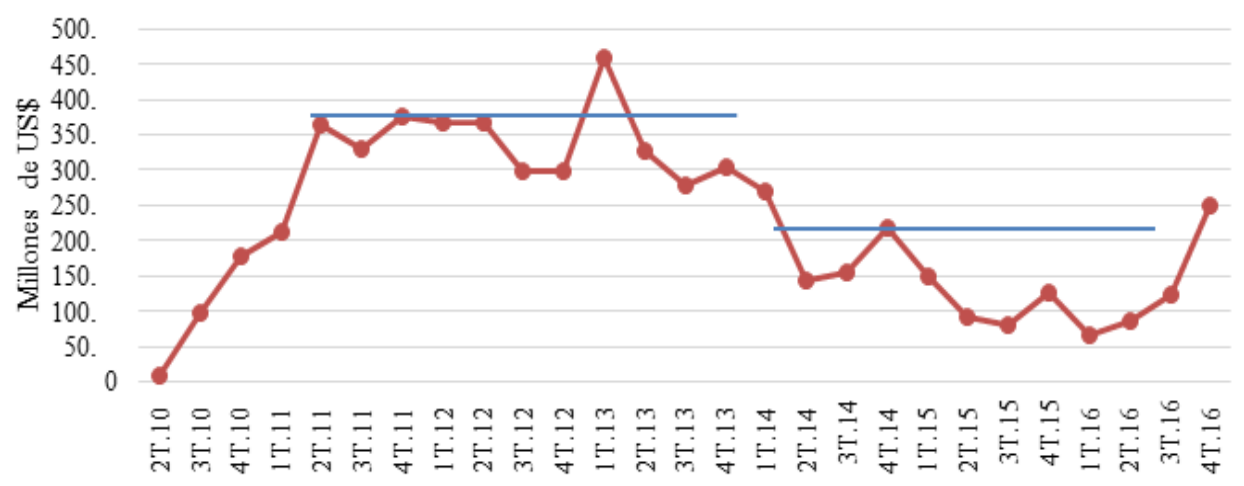

Figura 5. Valor trimestral de las exportaciones de GNL del Perú, período 2010-2016 Fuente: Gerencia Central de Estudios Económicos. BCRP

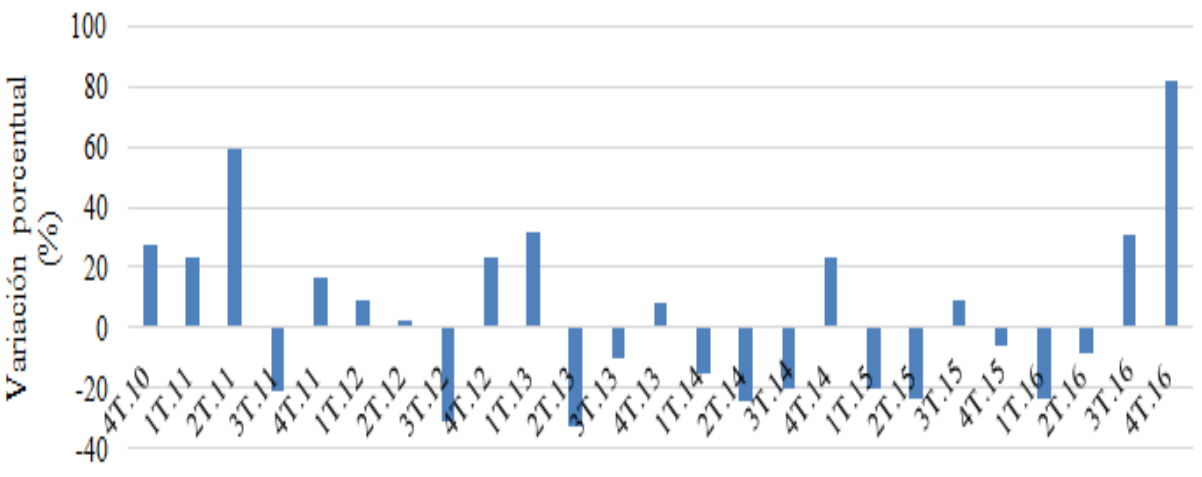

Figura 6. Variación porcentual del precio del GNL, período 2010-2016 Fuente: Gerencia Central de Estudios Económicos. BCRP

plazo y una tendencia ligeramente creciente en el mediano plazo, llegando al máximo volumen de exportación de 9,589 millones de $\mathrm{m}^{3}$ en el 2016 (Figura 7).

Cabe notar que el período 2011-2016, el volumen de las exportaciones anuales se mantuvo por encima de los 8 millones de $\mathrm{m}^{3}$, habiendo experimentado su menor valor en el $2015\left(8,093\right.$ millones de $\left.\mathrm{m}^{3}\right)$. Y mostró tasas de crecimiento fluctuantes: positivo en el 2011, 2013 y 2016; y negativo en el 2012, 2014 y 2015 (Tabla 2).

Tabla 2. Comportamiento del volumen de exportación de GNL del Perú, período 2010-2016

\begin{tabular}{lcc}
\hline & Volumen $\left(\right.$ miles de $\left.\mathrm{m}^{3}\right)$ & Variación porcentual $(\%)$ \\
\hline 2010 & $3.605,1$ & \\
2011 & $8.969,2$ & 148,8 \\
2012 & $8.737,5$ & $-2,6$ \\
2013 & $9.562,1$ & 9,4 \\
2014 & $9.226,6$ & $-3,5$ \\
2015 & $8.093,1$ & $-12,3$ \\
2016 & $9.589,0$ & 18,5 \\
\hline
\end{tabular}

En cuanto al volumen de las exportaciones trimestrales de GNL del Perú, mostró un comportamiento fluctuante en el corto plazo y una tendencia ligeramente creciente en el mediano plazo (Figura 8).
A partir del 4T-2010, el volumen de las exportaciones trimestrales ha estado por encima de los 2 millones de $\mathrm{m}^{3}$, con excepción de los trimestres 1T-2011, 2T-2014, 2T y 3T-2015, 1T-2016; pero nunca alcanzó los 3 millones de $\mathrm{m}^{3}$.

La evolución del volumen de las exportaciones trimestrales de GNL del Perú mostró un comportamiento estacional en el período 2011-2012, con tasas de crecimiento positivo en el tercer trimestre. En el período 2014-2016 cambió su comportamiento estacional, por ejemplo, mostró tasas de crecimiento negativo en $2 \mathrm{~T}$ del 2014-2015, en 1T del 2015-2016, y tasas de crecimiento positivo en 4T del 2014-2016 (Figura 4).

\section{Evolución del precio del GNL del Perú}

El precio del GNL tuvo un crecimiento significativo en el 2011, pasando de US $\$ 78 / \mathrm{m}^{3}$ (2010) a US $\$ 143,1 / \mathrm{m}^{3}$ (crecimiento del $82 \%$ ), similar nivel del precio se mantuvo en 2012-2013 (Figura 9, Tabla 3).

Pero en los años siguientes, el precio del GNL cae significativamente, llegando a US\$54,6/ $\mathrm{m}^{3}$ (2016), representando una caída del 64,15\% con respecto al 2012.

En cuanto al comportamiento trimestral del precio del GNL, mostró un comportamiento fluctuante en el corto plazo (Figura 10). Su tendencia fue positiva hasta el 1T- 


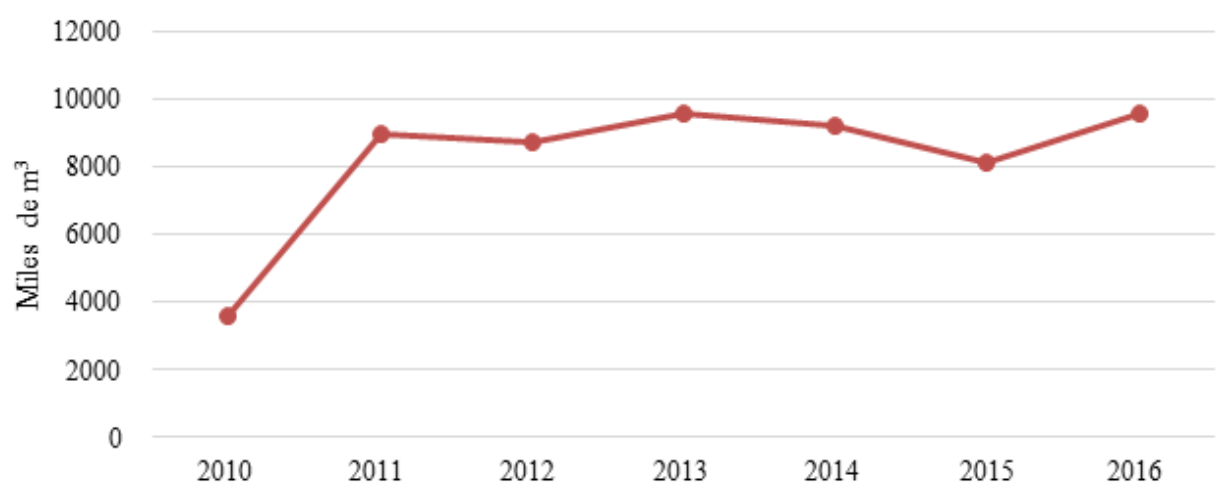

Figura 7. Volumen de las exportaciones de GNL del Perú, período 2010-2016 Fuente: Gerencia Central de Estudios Económicos. BCRP

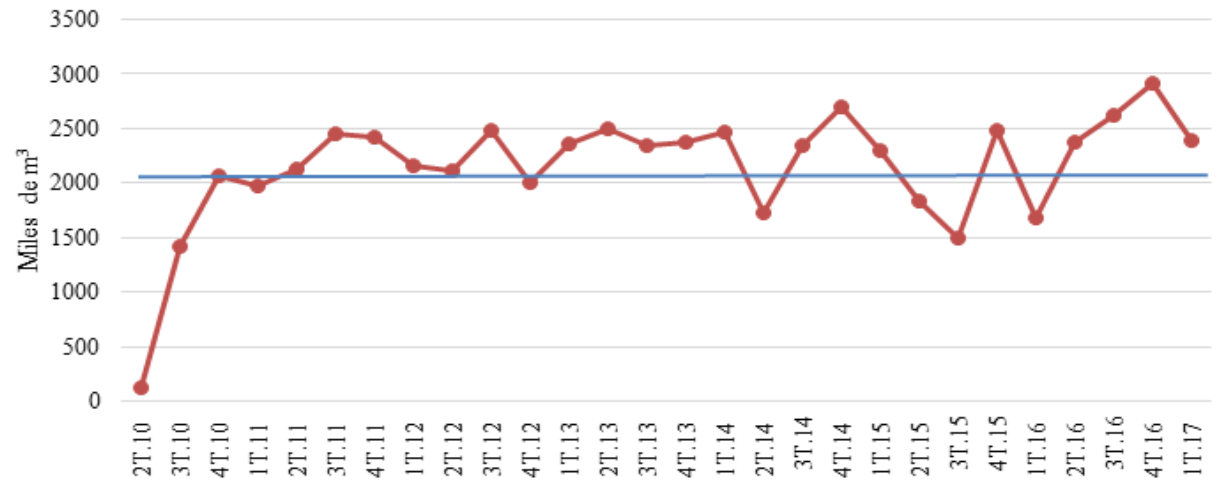

Figura 8. Volumen de las exportaciones trimestrales de GNL del Perú, período 2010-2016 Fuente: Gerencia Central de Estudios Económicos. BCRP

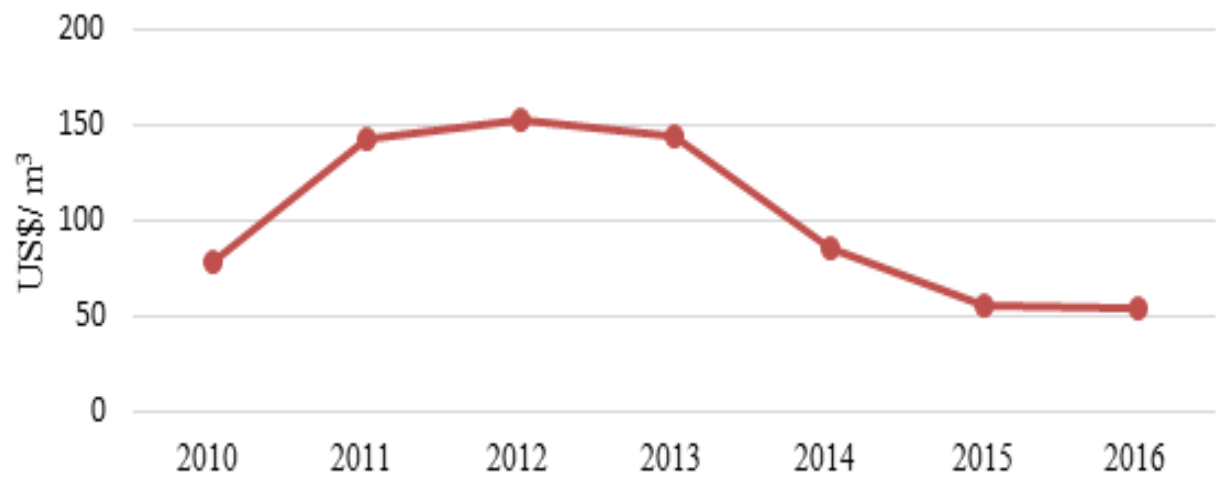

Figura 9. Precio del GNL del Perú, período 2010-2016

Fuente: Gerencia Central de Estudios Económicos. BCRP

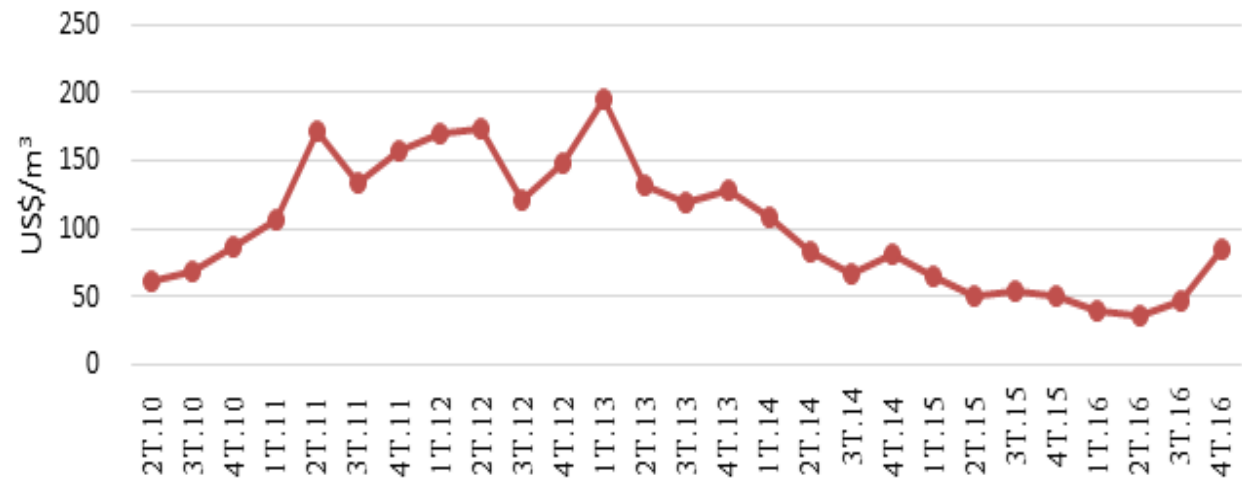

Figura 10. Precio del GNL del Perú, período 2010-2016

Fuente: Gerencia Central de Estudios Económicos. BCRP 
2013, llegando a su valor máximo de US\$195,67/m³. En los siguientes trimestres su tendencia ha sido negativa, llegando a su valor mínimo de U\$35,91/ $\mathrm{m}^{3}$ en el 2T-2016 (muy por debajo de lo alcanzado en 2T-2010). Para luego mostrar una recuperación en los siguientes trimestres (US\$85,48/m³ en 4T-2016).

Tabla 3. Comportamiento del precio del GNL peruano, período 2010-2016

\begin{tabular}{lcc}
\hline & Precio (US\$/m3) & Variación porcentual (\%) \\
\hline 2010 & 78,6 & \\
2011 & 143,1 & 82,0 \\
2012 & 152,3 & 6,4 \\
2013 & 143,5 & $-5,8$ \\
2014 & 85,2 & $-40,6$ \\
2015 & 55,5 & $-34,9$ \\
2016 & 54,6 & $-1,6$ \\
\hline
\end{tabular}

Cabe notar que cuando el precio del GNL está por encima de los $\$ 100 / \mathrm{m}^{3}$ (periodo del 1T-2011 al 1T-2014) muestra una mayor varianza $(721,66)$ pero un menor coeficiente de variación $(0,187)^{2}$, que cuando está por debajo de los $\$ 100 / \mathrm{m}^{3}$ (período del 2T-2014 al 4T-2016) con varianza: 280.21 y coeficiente de variación: 0.279 .

Cabe indicar que los meses de frio invierno en las regiones de Asía y Europa coinciden con el incremento del precio del GNL en el 4T del período 2010-2016 (con excepción del 2015).

En relación al precio del petróleo (US\$/barril), el precio del GNL $\left(\mathrm{US} \$ / \mathrm{m}^{3}\right)$ ha sido mayor y más fluctuante durante el 4T-2010 al 1T-2014 (coeficiente de variación: 0,2129), en cambio el precio del petróleo ha sido más estable (coeficiente de variación: 0,0728). Los mayores precios del GNL observados en los años 2011-2013 (Figura 16) se explicaría por el incremento del comercio mundial de GNL en los referidos años, con un nivel de importación promedio anual de $328 \mathrm{bmc}^{3}$, en comparación al promedio anual de 255,6 bmc del período 2008-2010. Los mayores precios se explicarían también por el aumento del consumo mundial de gas natural, que según el $\mathrm{BP}^{4}$ aumentó de $3.187,6$ bmc (2010) a 3.383,8 bmc (2013). La otra razón de los precios altos del GNL es por estar indexado al precio del petróleo, el que promediaba los US\$100/barril. Pero en el 2014, aún cuando la importación mundial de GNL aumentó a 333,3 bmc (2.45\% mayor al del 2013), el precio promedio anual de GNL disminuyó en $40,6 \%$ en relación al del 2013, coincidiendo con la caída continua del precio del petróleo a partir del 3T-2014.

En el resto del período (2T-2014 al 4T-2106) la diferencia de nivel entre ambos precios (GNL y petróleo) disminuyó significativamente (Figura 11), pero el GNL tuvo menor fluctuación (coeficiente de variación: 0,2794) que el petróleo (coeficiente de variación: 0,3754). La caída significativa del precio del GNL, alcanzado niveles similares al precio del petróleo, evidencia que en los últimos años está indexado al precio del petróleo en un mayor grado.

En el período 4T-2010 al 1T-2014 se observó una correlación positiva entre el precio de ambos combustibles (coeficiente de correlación: 0,731), pero disminuye en el período 2T-2014 al 4T-2016 (coeficiente de correlación: 0,6359). Esta relación positiva hace evidente que el incremento o disminución del precio del petróleo va acompañado con el incremento o disminución del gas natural, esto debido a que ambos son combustibles con cierto grado de sustitución (Figura 12), y que según López (2017) existen contratos de largo plazo con precios del GNL indexados al petróleo.

\section{Relación entre el volumen de exportación y precio del GNL del Perú}

En el período del 1T- 2011 al 1T-2014, cuando el precio del GNL estaba por encima de US $\$ 100 / \mathrm{m}^{3}$, el volumen de exportación presentó menores fluctuaciones (coeficiente de variación: 0,0782) en comparación a las que se presenta en el período del 2T-2014 al 1T-2017 (coeficiente de variación: 0,2) cuando el precio estaba por debajo de US\$100/ $\mathrm{m}^{3}$. Esto mostró que a precios elevados el volumen de exportación es menos sensible al precio, que cuando los precios son bajos (Figura 13). La razón sería que ante precios altos (por encima de US\$100/ $\mathrm{m}^{3}$ ), la subida o caída del precio no es un estímulo suficiente para variar significativamente el volumen de las exportaciones, pues se seguiría obteniendo niveles aceptables del valor de las exportaciones. Pero cuando el precio está por debajo de los US $\$ 100 / \mathrm{m}^{3}$, la caída del precio es un estímulo para disminuir el volumen de exportación e incrementar el volumen a destinarse al mercado interno, y ante un incremento del precio se destinaría un mayor volumen a las exportaciones.

En el período del 1T-2011 al 1T-2014, durante el cual el precio del GNL estuvo por encima de los US $\$ 100 / \mathrm{m}^{3}$, el coeficiente de correlación entre las series trimestrales del precio y volumen de exportación del GNL fue de -0,246, evidenciando una relación inversa entre ambas variables

En cambio, en el periodo del 2T-2014 al 1T-2015, cuando el precio cae por debajo de los US\$100/ $\mathrm{m}^{3}$, el coeficiente de correlación fue de 0,313. Lo cual indica que una caída o aumento del precio conlleva a una caída o aumento del volumen de las exportaciones.

En la Figura 14, se observa que el periodo del 1T-2011 al 1T-2014, el signo de la variación porcentual (positivo o negativo) del volumen de exportación y del precio coincide en 4 de 13 trimestres, en cambio, en el periodo del 2T-2014 al 1T-2015 coincide en 7 de los 11 trimestres, evidenciado una mayor correlación positiva. 


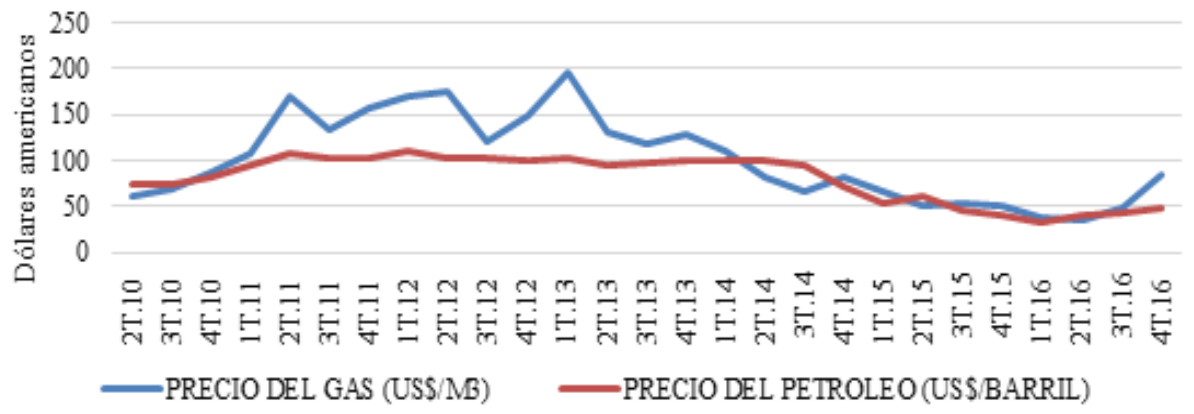

Figura 11. Precio del GNL del Perú y el precio del petróleo, período 2010-2016 Fuente: Gerencia Central de Estudios Económicos. BCRP

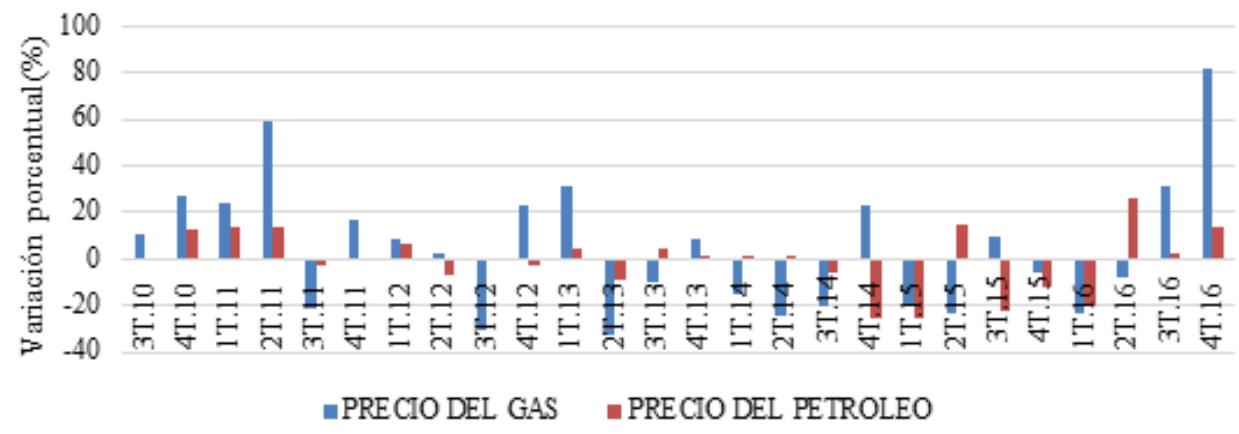

Figura 12. Variación porcentual del precio de GNL y petróleo, período 2010-2016 Fuente: Gerencia Central de Estudios Económicos. BCRP

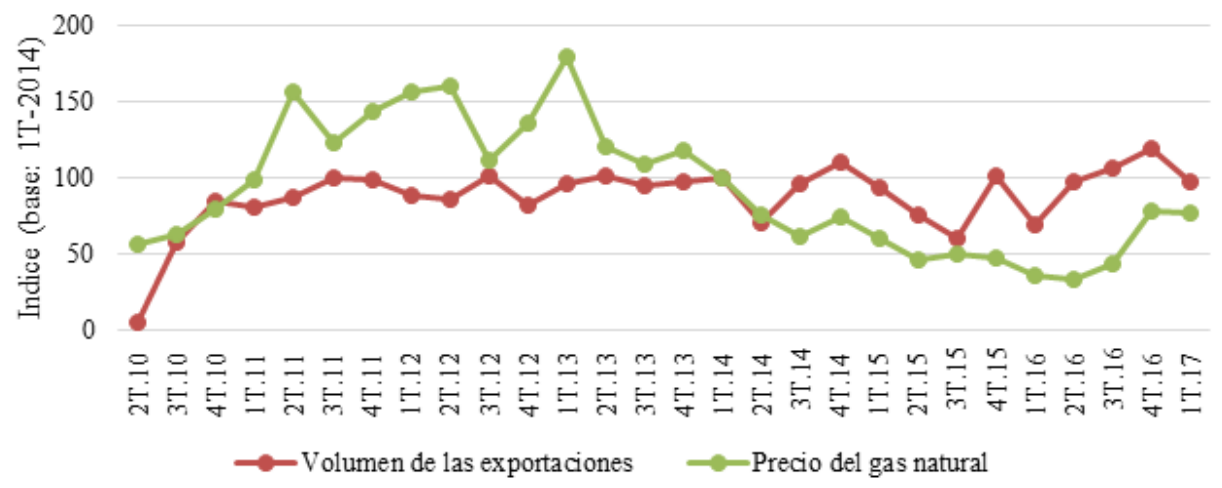

Figura 13. Índices del volumen de exportaciones y precio del GNL. período 2010-2016 Fuente: Gerencia Central de Estudios Económicos. BCRP

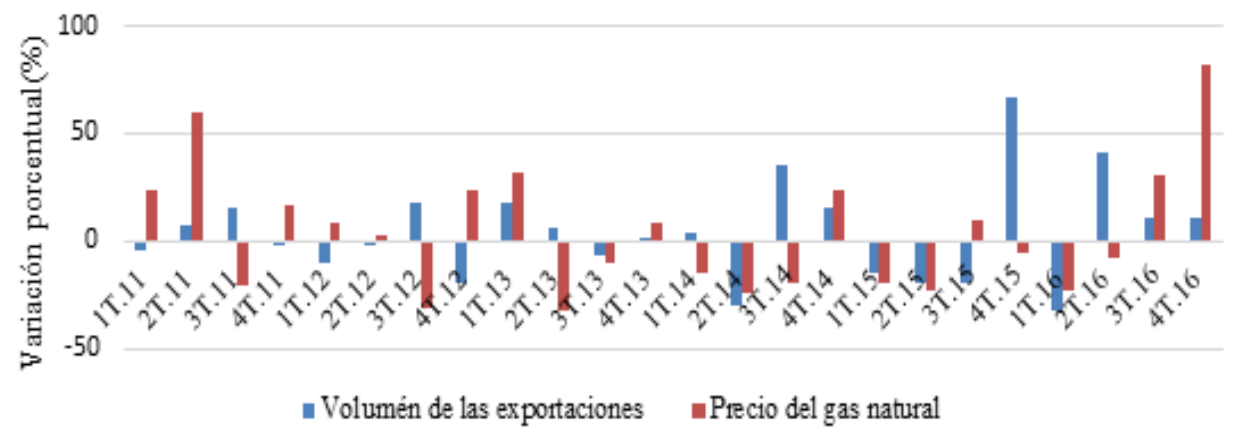

Figura 14. Variación porcentual del volumen de exportación y precio del GNL del Perú Fuente: Gerencia Central de Estudios Económicos. BCRP 


\section{Relación entre el valor de las exportaciones, volumen y precio del GNL del Perú}

Si bien el valor de las exportaciones del GNL es el resultado del producto del volumen de exportación y precio, cabe notar que cuando el precio del GNL está por encima de $\$ 100 / \mathrm{m}^{3}$ (1T-2011 al 1T-2014) el comportamiento del índice del precio es muy similar al comportamiento del índice del valor de las exportaciones (coeficiente de correlación: 0,9172), Figura 15. Igual comportamiento se observó cuando el precio está por debajo de $\$ 100 / \mathrm{m}^{3}$ (2T2014 al 1T-2017), pero en un menor grado (coeficiente de correlación: 0,8618 ).

En base al coeficiente de correlación entre los índices (Tabla 4), cabe indicar que en el período 2T-2011 al 4T2016 la similitud del comportamiento del volumen con el valor de las exportaciones aumentó (coeficiente de correlación: 0,7338) con respecto al período anterior (coeficiente de correlación: 0,1546).

Por tanto, los precios altos del GNL, tienen influencia significativamente en el comportamiento del valor de las exportaciones de gas natural, pero con precios bajos el volumen de las exportaciones incrementa su influencia.
Tabla 4. Coeficientes de correlación entre índices del valor y volumen de exportación, y precio del GNL, período 2010-2016

\begin{tabular}{lcc}
\hline $\begin{array}{l}\text { Coeficiente de } \\
\text { correlación entre } \\
\text { indices del: }\end{array}$ & 1T-2011 al 1T-2014 & 2T-2014 al 4T-2016 \\
\hline $\begin{array}{l}\text { Valor y } \\
\text { volumen de las } \\
\text { exportaciones }\end{array}$ & 0,1546 & 0,7338 \\
$\begin{array}{l}\text { Valor de las } \\
\text { exportaciones y } \\
\text { precio }\end{array}$ & 0,9172 & 0,8618 \\
\hline
\end{tabular}

\section{Mercado mundial del gas natural licuado.}

Según el Observatory of Economic Complexity, en el 2016 el valor de la importación mundial de GNL fue US\$ 73.100 millones, estando en primer lugar Japón (41,3\%), seguido de Corea del Sur (16,7\%), China $(12,2 \%)$, India $(7,4 \%)$ y España (3,9\%). La importación mundial de GNL se incrementó en el 2010-2014, pasando de US\$ 103.000 millones (2010) a US\$174.000 millones (2014). Pero disminuyó significativamente en los siguientes años: US\$ 113.000 millones (2015) y US\$ 73.100 (2016), Figura 16.

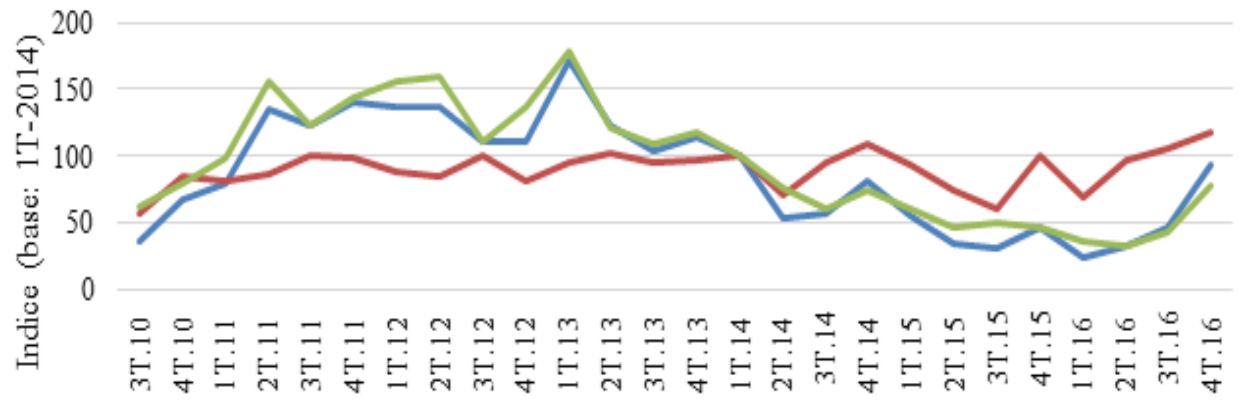

-Valor de las exportaciones _-Volumen de las exportaciones _-Precio del gas natural

Figura 15. Indices del valor, volumen de exportaciones y precio del GNL peruano, período 2010-2016 Fuente: Gerencia Central de Estudios Económicos. BCRP

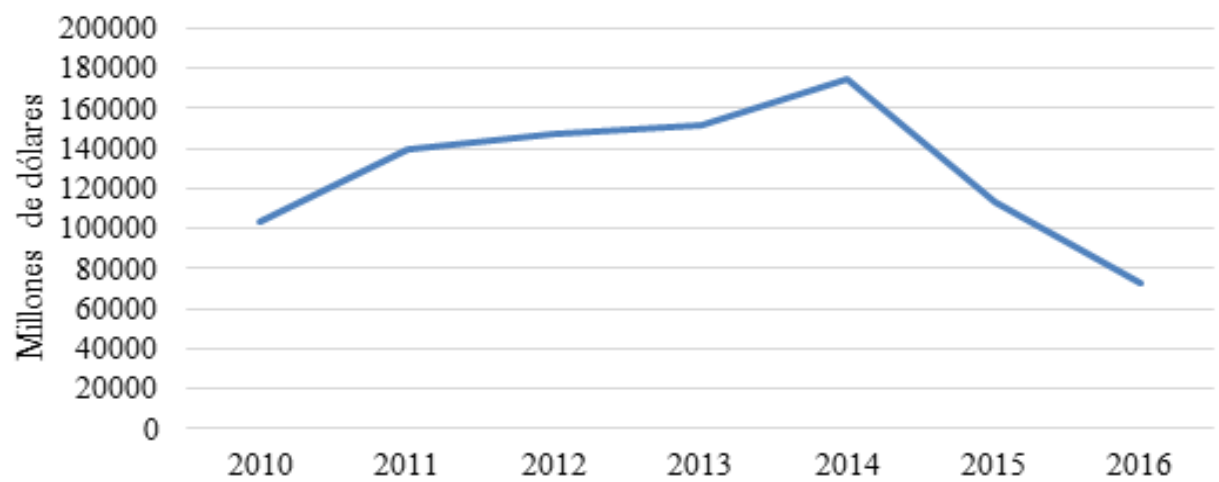

Figura 16. Importación mundial de GNL, período 2010-2016 Fuente: The Observatory of Economic Complexity (OEC) 
Países de destino de las exportaciones de GNL del Perú

Durante el 2010-2016 se identificaron al menos 13 países de destino de las exportaciones de GNL peruano, de los cuales tres países mostraron una demanda continua (España, México y Japón), habiendo sido los mayores importadores del GNL peruano. Y en 2015-2016 reaparecen Corea del Sur y China importando por un valor mayor al de Japón (Tabla 5).

En el 2016, en cuanto a la participación porcentual como destino de las exportaciones del GNL peruano, España ocupa el primer lugar (40,85\%), seguido de México (26,15\%), Corea del Sur (10,21\%), China (8,76\%) y Japón (7,23\%). Cabe notar que la participación de los referidos países ha sido fluctuante en el período 2010-2016 (Tabla 6).

Según Osinergmin (s.f.) la razón de la baja demanda del GNL peruano por países de Europa, es que ellos han sido abastecidos tradicionalmente mediante ductos en vez de GNL.

Siendo España el principal mercado de destino del GNL peruano, este significó el $13 \%$ del valor total de sus importaciones de GNL en el 2016, siendo sus principales proveedores de GNL: Nigeria (30\%), Argelia (29\%) y Qatar (7,3\%). En el caso de México significó el 27\%, siendo su principal proveedor EEUU (32\%). En el caso de China significó el 1,2\%, siendo sus principales proveedores: Australia (42\%), Qatar (23\%), Indonesia (10\%) y Malasia (9,2\%). En el caso de Corea del Sur significó el 0,32\%, siendo sus principales proveedores: Qatar (36\%), Australia (15\%), Oman (13\%), Malasia (11\%) e Indonesia (11\%). Y en el caso de Japón significó el 0,1\%, siendo sus principales proveedores: Australia (28\%), Malasia (18\%), Qatar (13\%), Indonesia $(8,6 \%)$, Rusia $(8,3 \%)$ y Emiratos Árabes $(5,6 \%)$.

La razón principal que explicaría la baja participación del GNL del Perú en el total de las importaciones de España, China, Japón y Corea del Sur, es la menor distancia que existe entre los referidos países y sus principales proveedores, puesto que algunos de los principales países exportadores de gas natural a nivel mundial se encuentran en África, Medio Oriente y Asia. En el caso de México, también el factor distancia ha sido preponderante, toda vez que México colinda con los EEUU.

El valor de las importaciones totales de GNL por parte de Japón, Corea del Sur y China, en general han mostrado una tendencia creciente en el período 2011-2014 (siendo más significativa para Japón y Corea del Sur). Luego esta tendencia cambió a decreciente en los años 2015 y 2016, (Figura 17).
Tabla 5. Valor de las exportaciones de GNL peruano por países de destino, período 2010-2016 (millones de dólares)

\begin{tabular}{lrrrrrrr}
\hline Paises & 2010 & 2011 & 2012 & 2013 & 2014 & 2015 & 2016 \\
\hline Belgica-Luxemburgo & 17,7 & 17,8 & & & & & \\
Brasil & 36,2 & & & & & & \\
Canadá & 12,6 & & & & & & \\
China & 23,3 & 38,7 & & & & & 45,9 \\
Corea del Sur & 44,1 & 243,0 & & 293,0 & & 10,9 & 53,5 \\
EEUU & 66,3 & 121,0 & & & & & \\
España & 102,0 & 496,0 & 695,0 & 535,0 & 294,0 & 153,0 & 214,0 \\
Francia & & & & & 16,0 & 46,2 & 15,5 \\
India & & & & & & 13,6 & 10,2 \\
Italia & & & & & & & 9,9 \\
Japón & & 224,0 & 472,0 & 363,0 & 55,3 & 43,9 & 37,9 \\
México & 26,5 & 77,1 & 82,5 & 215,0 & 463,0 & 209,0 & 137,0 \\
Tailandia & & 167,0 & 146,0 & & & & \\
Otros países de Asia & & 30,9 & & & & & \\
TOTAL & & & & & & & \\
\hline
\end{tabular}

TOTAL

$328,7 \quad 1.415,51.395,5 \quad 1.406,0 \quad 828,3 \quad 476,6 \quad 523,9$

Fuente: The Observatory of Economic Complexity (OEC)

Tabla 6. Participación porcentual en el destino de las exportaciones de

\begin{tabular}{lrrrrrrr}
\hline Paises & 2010 & \multicolumn{1}{c}{2011} & 2012 & 2013 & 2014 & 2015 & 2016 \\
\hline Bélgica-Luxemburgo & 5,38 & 1,26 & & & & & \\
Brasil & 11,01 & & & & & & \\
Canadá & 3,83 & & & & & & \\
China & 7,09 & 2,73 & & & & & 8,76 \\
Corea del Sur & 13,42 & 17,17 & & 20,84 & & 2,29 & 10,21 \\
EEUU & 20,17 & 8,55 & & & & & \\
España & 31,03 & 35,04 & 49,80 & 38,05 & 35,49 & 32,10 & 40,85 \\
Francia & & & & & 1,93 & 9,69 & 2,96 \\
India & & & & & & 2,85 & 1,95 \\
Italia & & & & & & & 1,89 \\
Japón & & 15,82 & 33,82 & 25,82 & 6,68 & 9,21 & 7,23 \\
México & 8,06 & 5,45 & 5,91 & 15,29 & 55,90 & 43,85 & 26,15 \\
Tailandia & & 11,80 & 10,46 & & & & \\
Otros países de Asia & & 2,18 & & & & & \\
Total & 100,00 & 100,00 & 100,00 & 100,00 & 100,00 & 100,00 & 100,00 \\
\end{tabular}

El comportamiento observado del valor anual de las importaciones totales de GNL de España, México, Japón y Corea del Sur (principales mercados de destino del GNL del Perú), coincide con el comportamiento del valor de las exportaciones del GNL peruano a los referidos países, mostrando sus mayores valores en el período 2011-2013, y disminuyendo en el período 2014-2016 (con excepción de México que creció en el 2014). Lo observado evidencia la influencia de las importaciones totales de los referidos países en el valor de las exportaciones de GNL del Perú (Figura 18).

A pesar que el valor de las importaciones totales de GNL por parte de Japón, Corea del Sur y España han disminuido en el 2016 (Tabla 7), con excepción de China, sus niveles 
de importación siguen siendo significativos, lo que garantiza la posibilidad de incrementar el volumen y valor de las exportaciones de GNL peruano.

Considerando los volúmenes importados del GNL peruano, los mercados que se mantienen relevantes son México y España (Tabla 8).
Tabla 7. Valor de las importaciones totales de GNL por países (millones de dólares)

\begin{tabular}{cccccccc}
\hline PAISES & 2010 & 2011 & 2012 & 2013 & 2014 & 2015 & 2016 \\
\hline España & 6.960 & 7.790 & 7.100 & 6.220 & 5.770 & 3.000 & 2.840 \\
México & 874 & 621 & 178 & 1.350 & 1.650 & 732 & 784 \\
Japón & 36.500 & 55.700 & 68.700 & 67.000 & 70.700 & 42.400 & 30.200 \\
Corea del Sur & 15.700 & 21.900 & 25.000 & 28.100 & 30.600 & 18.000 & 12.200 \\
China & 2.700 & 5.110 & 6.540 & 9.350 & 11.900 & 8.370 & 8.940 \\
\hline Fuente: The Observatory of Economic Complexity (OEC)
\end{tabular}

Tabla 8. Volumen importado de GNL peruano por países (billones de metros cúbicos)

\begin{tabular}{lccc}
\hline & 2015 & 2015 & 2016 \\
\hline China & & & 0,3 \\
Corea del Sur & & & 0,2 \\
España & 1,2 & 0,9 & 1,7 \\
Francia & 0,1 & 0,3 & 0,2 \\
India & & 0,1 & 0,1 \\
Japón & 0,1 & 0,2 & \\
México & 4,3 & 3,5 & 2,9 \\
\hline
\end{tabular}

Fuente: BP Statistical Review of World Energy 2016, 2017
Se debe consolidar y aumentar las exportaciones hacia Corea del Sur y Japón, aun cuando en el 2016 demandaron el $17.44 \%$ de las exportaciones de GNL peruano, según Lopez (2017) ambos países concentran el 50\% de la demanda mundial de GNL.

\section{Conclusiones}

Durante el período 2010-2016, el valor de las exportaciones de gas natural licuado (GNL) del Perú ha sido determinado principalmente por la demanda de España, México, Japón y Corea del Sur, que representaron el $84.4 \%$ en el 2016 . En el corto plazo el valor de las exportaciones de GNL

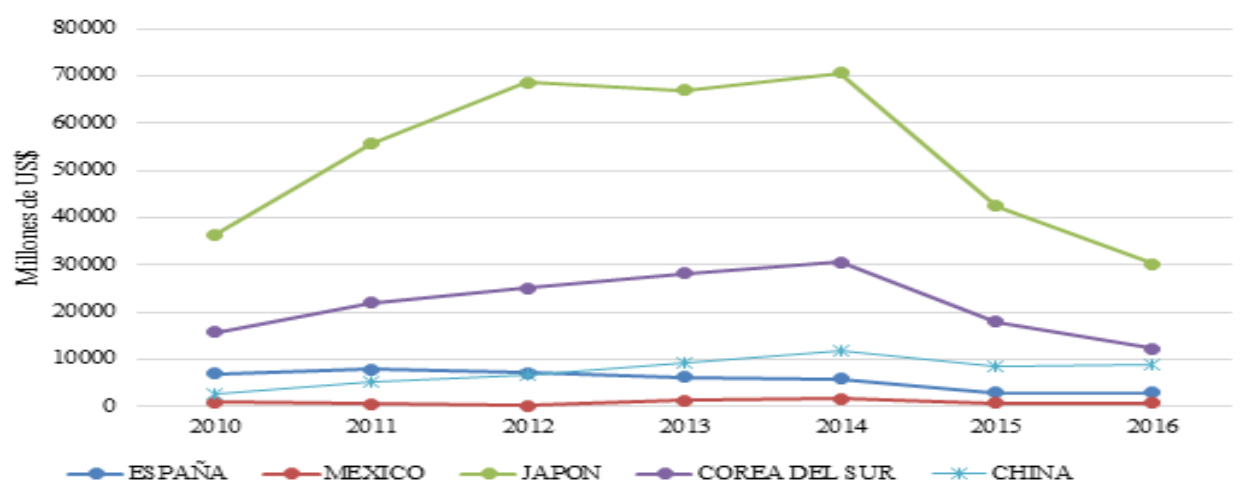

Figura 17. Valor de las importaciones totales de GNL de España, México, Japón, Corea del Sur y China, período 2010-2016

Fuente: The Observatory of Economic Complexity (OEC)

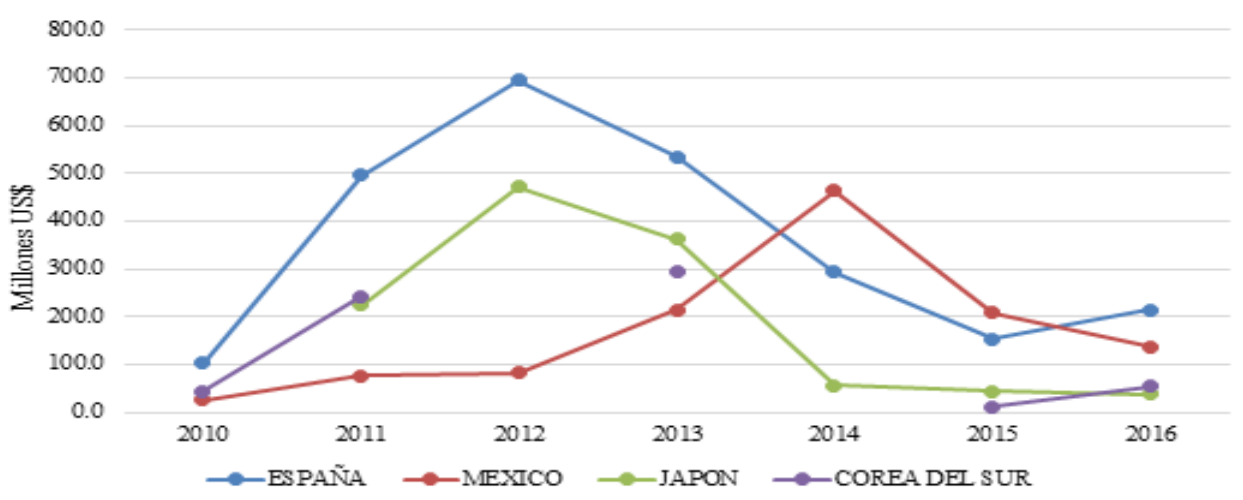

Figura 18. Valor de las exportaciones de GNL peruano hacia España, México, Japón y Corea del Sur, período 2010-2016

Fuente: The Observatory of Economic Complexity (OEC) 
peruano ha evidenciado un comportamiento estacional, mostrando tasas de crecimiento positivo en el cuarto trimestre, coincidiendo con la estación de invierno de los cuatro países mencionados. Similar comportamiento estacional se observa en el volumen de exportación y precio del GNL. El elevado nivel del valor de las exportaciones de GNL peruano en el período 2011-2013, que en promedio anual alcanzó US\$1.328,6 millones, se explica principalmente por el aumento de su precio, cuyo valor promedio anual estuvo por encima de los US\$140/ $\mathrm{m}^{3}$. Y el bajo nivel del valor de las exportaciones de GNL peruano en el período 2014-2016, que en promedio anual fue de US\$586.2 millones, se explica por la caída significativa de su precio, que en el referido período alcanzo un valor promedio anual de US $\$ 65.1 / \mathrm{m}^{3}$, muy por debajo de los US\$146,2/m3 alcanzado en el período 2011-2013. Lo que evitó una mayor caída del valor de las exportaciones de GNL peruano en el período 20142016, fue que el volumen de exportación promedio anual (8.969,5 miles de metros cúbicos) mantuvo un nivel cercano al del período 2011-2013 (9.088,6 miles de metros cúbicos). Aun cuando actualmente el precio de GNL se encuentra en niveles bajos, el valor de las exportaciones de GNL peruano puede incrementarse si se logra aumentar el volumen de exportación hacia China, cuya importación en volumen a nivel mundial se incrementó en $30 \%$ en el 2016 , y ha empezado a nuevamente a adquirir el GNL peruano. España se presenta como un mercado en crecimiento, pues el volumen importado de GNL peruano aumentó en $88.8 \%$ el 2016. Y Corea del Sur y Japón también son importantes mercados potenciales para el GNL peruano, pues en el 2016 adquirieron el 43,9\% del volumen de la importación mundial de GNL.

\section{Literatura citada}

Banco Central de Reserva del Perú (BCRP). S.f. Recuperado de: http://www.bcrp.gob.pe/

The Observatory of Economic Complexity [OEC] s.f. Recuperado de : https://atlas.media.mit.edu/en/

BP Statistical Review of World Energy. 2017. Recuperado de: https://www.bp.com/content/dam/bp/en/corporate/ $\mathrm{pdf} /$ energy-economics/statistical-review-2017/bpstatistical-review-of-world-energy-2017-full-report.pdf

Gerencia de Fiscalización de Gas Natural. 2014. Presentación: Mercado Internacional del Gas Natural. Osinergmin. Recuperado de: http://www. osinergmin.gob.pe/newweb/uploads/Publico/ OficinaComunicaciones/EventosRealizados/ ForoTacna/3/2-Mercado $\% 20$ Internacional $\% 20 \mathrm{de} \% 20$ GN-Gerardo\%20Meza.pdf

López, P. 2017. La península ibérica como hub de gas natural licuado. Revista ICE. No. 895.

http://www.revistasice.com/CachePDF/ICE 895 6172 66226F2516CA51F144CD01FA6DAD2CAF.pdf

Observatorio Energético Minero. Osinergmin. s.f. Recuperado de: http://observatorio.osinergmin.gob.pe/ 